\title{
Composición taxonómica y aspectos zoogeográficos de peces de profundidad (90-540m) del Golfo de California, México
}

\author{
Juana López-Martínez ${ }^{1}$, Alejandro Acevedo-Cervantes ${ }^{2}$, Eloisa Herrera-Valdivia ${ }^{1}$, Jesús \\ Rodríguez-Romero ${ }^{1} \&$ Deivis S. Palacios-Salgado ${ }^{1}$ \\ 1. Centro de Investigaciones Biológicas del Noroeste S.C. Km. 2.35 Carretera a Las Tinajas, S/N Colonia Tinajas, \\ Guaymas, Sonora, México C. P. 85460; jlopez04@cibnor.mx, eloisa04@cibnor.mx, jrodri04@cibnor.mx, \\ deivis23@yahoo.com \\ 2. Instituto Tecnológico de Guaymas, Km cuatro Carretera al Varadero s/n, Las Playitas Guaymas Sonora, México; \\ aleacevedo@yahoo.com
}

Recibido 26-I-2011. Corregido 09-VI-2011. Aceptado 07-VII-2011.

\begin{abstract}
Taxonomic composition and zoogeographical aspects of deep sea fishes $(\mathbf{9 0 - 5 4 0 m})$ from the Gulf of California, Mexico. The Gulf of California has a high variety of ecosystems that allow different services and the fishery resources play a prominent role in its ecology, evolution and economics. Fish coastal species have been previously reported for most coastal areas, especially those species that are subject to fishing, however, little is known on the species from deep sea zones, due to sampling difficulties. We studied the deep sea fishes collected with trawl nets during three research surveys in the Gulf of California, Mexico in 2004-2005. We provide a systematic checklist and some notes on biogeographical aspects. For this, 74 fishing hauls were done, and a total of 9898 fishes were captured, belonging to two classes, 15 orders, 35 families, 53 genera and 70 species. The best represented families in number of species were: Paralichthyidae (eight), Serranidae (six), and Scorpaenidae and Triglidae with five species each one. The typical families from deep waters were: Ophidiidae, Moridae, Lophiidae, Scorpaenidae, Triglidae, Paralichthydae, Pleuronectidae and Cynoglossidae. Size range varied from $13 \mathrm{~cm}$ for the Splinose searobin (Bellator xenisma) to $234 \mathrm{~cm}$ in the Pacific Cutlassfish (Trichiurus nitens). The biogeographical affinity showed that species with affinity to the East Tropical Pacific (ETP) dominated, followed by species from San Diego-Panamic, San Diego-Panamic-Peruvian-Chilean and Oregonian-Cortes provinces, respectively. A biogeographic overlap was found in the fauna, which reflects the Gulf of California's geographical position, with distribution limits of species from temperate, tropical and warm-temperature transition affinities, divisions that characterize the Gulf of California. Taxonomic status of fish with a focus on composition, location, characterization and zoogeography are fundamental to any subject of biodiversity and fisheries management actions. Rev. Biol. Trop. 60 (1): 347-360. Epub 2012 March 01.
\end{abstract}

Key words: biogeography, systematic checklist, deep fishes, Gulf of California.

El Golfo de California es un mar marginal con características especiales que le permiten tener una amplia gama de ecosistemas que dan como resultado una amplia variedad ecológica de fauna y flora marina, donde los peces resaltan ampliamente por su gran biodiversidad (Pérez-España et al. 1996, Thomson et al. 2000). Los estudios realizados en éste golfo son extensos (Chávez \& Arvizu-Martínez 1972, Chávez 1986, Thomson \& Mckibbin 1978,
Amezcua-Linares 1985, Pérez-Mellado \& Findley 1985, Van Der Heiden 1985, RodríguezRomero 1992, 1994, 2008, Pérez-España et al. 1996, Rodríguez-Romero et al. 1998, Thomson et al. 2000). Los listados sistemáticos han representado una herramienta necesaria para la investigación de los ecosistemas (RodríguezRomero 1992, 1994, 2008, Abitia-Cárdenas et al. 1994, De la Cruz-Agüero et al. 1994, Castro-Aguirre et al. 1995, Galván-Magaña et al. 
1996, 2000, Moncayo-Estrada et al. 2006), ya que son el punto de partida de cualquier estudio comparativo en dos períodos de tiempo.

La mayoría de los estudios ictiofaunísticos efectuados en el Golfo de California han sido realizados en ecosistemas costeros, especialmente en sistemas estuarino-lagunares y, apoyados principalmente de recolectas o de las capturas de la pesca comercial, quedando de lado los peces que no son accesibles a la pesca. Son escasos los trabajos de peces de profundidad, aun en el ámbito internacional (Arana et al. 2002, Powell et al. 2003, Gutiérrez-García 2004, Reyes 2007) y en particular en el Golfo de California existen los trabajos efectuados por Garman (1899), Goode \& Bean (1895), Grey (1956), Brewer (1973), Castro-Aguirre (1991) y otros, donde se hace una revisión de los registros de peces de profundidades mayores de los $90 \mathrm{~m}$.

La estructura de las comunidades de peces de profundidad es compleja, según AcevedoCervantes et al. (2009), puede estar formada por especies tanto de ámbito costero como de la zona profunda del talud continental formando ecotonos. A nivel mundial, algunas comunidades de peces demersales de aguas profundas son objeto de pesca comercial (Koslow et al. 2001, Gordon et al. 2003) y en muchos casos el conocimiento científico sobre ellas es escaso, se requiriere información a nivel de ecosistema y biología poblacional, con la finalidad de llegar a un manejo sustentable de estos recursos (Koslow et al. 2001, Gordon et al. 2003, Fossen et al. 2008).

Existe controversia sobre el grado de diversidad en ambientes marinos profundos, algunos autores han planteado una alta diversidad (Castro-Aguirre \& Balart 1996, Grassle 1989 , Grant 2000) y se sugieren diversos mecanismos para explicarla, tales como el efecto de la interacción de competencia y depredación (Grant 2000). Igualmente se ha propuesto que existe una clara diferencia entre la fauna de la plataforma continental y del talud continental y que las variables que influyen en el cambio de las comunidades de peces de profundidad son difíciles de establecer, aludiendo gradientes de factores como nivel de luz, sustrato y sedimentos, así como temperatura, oxígeno disuelto y salinidad (Powell et al. 2003, Fujita et al. 1995, Acevedo-Cervantes et al. 2009).

El objetivo de esta investigación es incrementar el conocimiento de la biodiversidad íctica, a partir de la composición taxonómica, aspectos ecológicos y zoogeográficos de los peces del Golfo de California que habitan en profundidades entre $90-540 \mathrm{~m}$, debido a que esta información es relevante en el contexto ecológico, pesquero y de conservación y/o manejo, así mismo, como punto de comparación para investigaciones posteriores.

\section{MATERIALES Y MÉTODOS}

Las muestras se obtuvieron de tres cruceros exploratorios realizados a bordo del buque de investigación BIP XII durante septiembre 2004, y febrero y mayo 2005. Los lances se efectuaron siguiendo un muestreo sistemático en la costa oriental del Golfo de California desde el sur de Puerto Peñasco Sonora, hasta Topolobampo en Sinaloa (Fig. 1), cada lance se efectuó con una duración de una hora de arrastre efectivo. El equipo de pesca utilizado fue una red de arrastre de fondo construida con material de polietileno trenzado de abertura de malla de una pulgada, de tamaño de $38 / 34$ m y perímetro de la boca en paño estirado de $68 \mathrm{~m}$, con una relinga superior de $38 \mathrm{~m}$. La red operó por popa a profundidades entre 90 y $540 \mathrm{~m}$, a una velocidad de $5.5 \mathrm{~km} / \mathrm{h}$. La captura una vez subida a bordo fue vaciada en la cubierta del barco, y de cada lance se tomó una muestra de $20 \mathrm{~kg}$ al azar, y se conservó en congelación hasta su posterior identificación en el laboratorio.

De la muestra obtenida, los peces fueron separados e identificados hasta especie a través de las claves de Jordan \& Evermann (18961900), Meek \& Hildebrand (1923-1928), Miller \& Lea (1972), Eschmeyer et al. (1983), Fischer et al. (1995) y Robertson \& Allen (2002). Para algunos grupos se utilizó bibliografía especializada, Castro-Aguirre \& Espinosa-Pérez (1996) para rayas, Espinosa-Pérez et al. (2004) 


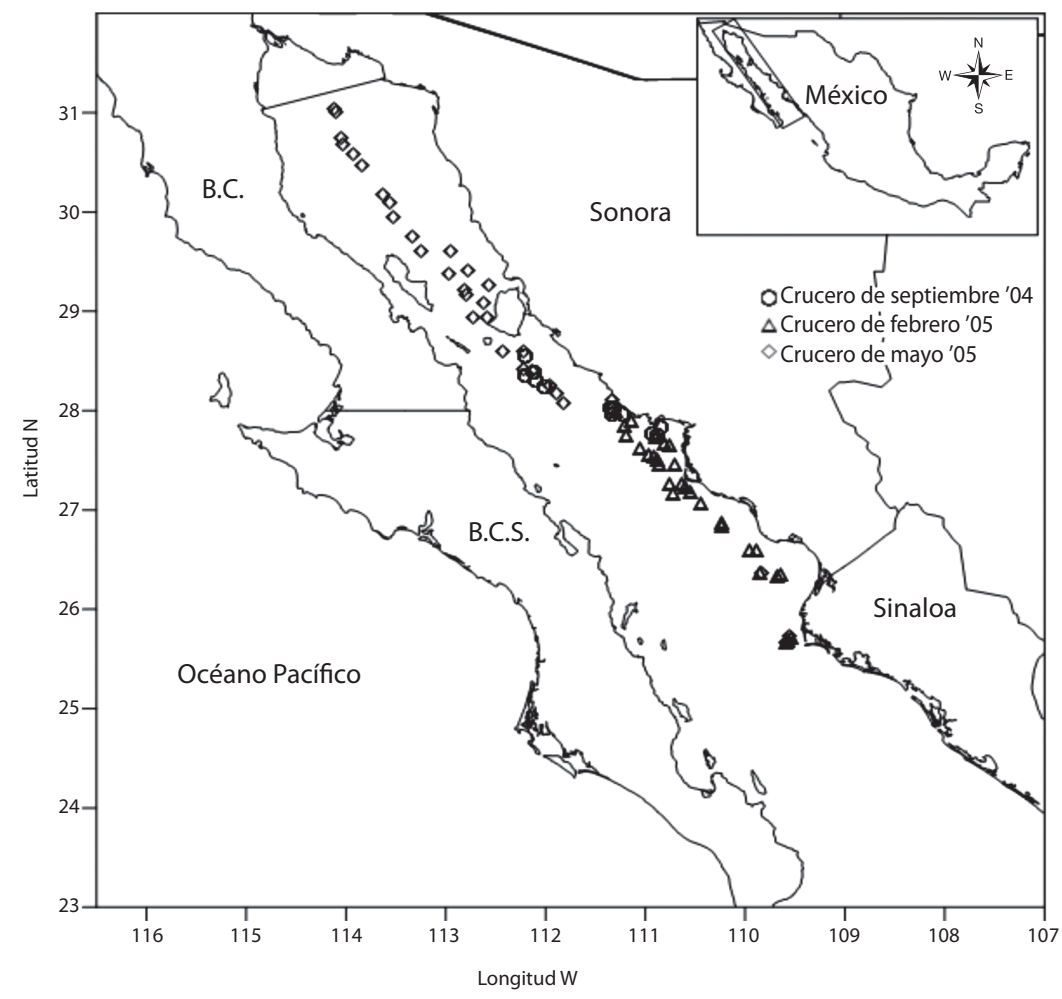

Fig. 1. Área de estudio de los peces de profundidad (90-540m) en el Golfo de California, México.

Fig. 1. Study Area fish deep (90-540m) in the Gulf of California, Mexico.

para tiburones, McPhail (1958) para la familia Sciaenidae, Ginsburg (1958) para Pleuronectiformes, Rosenblatt \& Johnson (1974) para Diplectrum, Walter \& Rosenblatt (1988) para Porichthys, Orr et al. (2000) y Compagno (1984) para tiburones. Ejemplares de referencia fueron fijados con formaldehído al $10 \%$ y posteriormente preservados en alcohol etílico al $70 \%$. Este material se encuentra en el laboratorio de pesquerías de la Unidad Guaymas del Centro de Investigaciones Biológicas del Noroeste, S.C. para ser incorporado a la Colección Ictiológica del Centro. De cada ejemplar se tomó la longitud total y estándar mediante un ictiómetro de $1 \mathrm{~mm}$ de precisión; el peso mediante una balanza de $0.1 \mathrm{~g}$ de precisión.

Con la finalidad de ubicar afinidad biogeográfica y nivel trófico de cada especie, se efectuó una revisión exhaustiva de bases de datos especializados en peces, tales como FISHBASE, ITIS, Discover Life, así como las claves de Robertson \& Allen (2002). El análisis de afinidad zoogeográfica fue determinada de acuerdo con el esquema básico de Briggs (1974, 1995), con modificaciones de Boschi (2000), Galván et al. (2000), Hastings (2000), Robertson \& Allen (2002), Robertson et al. (2004) y Horn et al. (2006) considerando las siguientes divisiones: PA: Provincia Aleutiana de Isla Nunivak en la Península de Alaska a Puget Sound en Washington, ésta es una provincia de aguas templado-frías; PO: Provincia Oregoniana se extiende de Puget Sound a Punta Concepción en California, la porción sur es considerada una zona de transición entre esta provincia y la de San Diego; PS: Provincia de San Diego de Punta Concepción a Bahía Magdalena en el sur de Baja California, corresponde 
a aguas templado-cálidas; PC: Provincia de Cortés (sinuscaliforniana) incluye la parte sur de Bahía Magdalena, y el Golfo de California entero; PM: Provincia Mexicana incluye la costa de México desde Mazatlán, Sinaloa, hasta el Istmo de Tehuantepec, Oaxaca; PP: Provincia Panámica se extiende hacia el sur desde El Salvador hasta alrededor de Cabo Blanco, en la parte norte del Perú; POT: Incluye especies de amplia distribución en el Pacífico Oriental Tropical, esta región incluye la costa oeste del continente americano entre $25^{\circ} \mathrm{N}$ en la parte sur de Bahía Magdalena, hasta los $5^{\circ} \mathrm{S}$ en Cabo Blanco parte norte de Perú; CT: Circumtropicales son las especies ícticas de amplia distribución en los mares tropicales del mundo.

El arreglo sistemático se realizó siguiendo los criterios de Nelson (2006). El arreglo de los géneros y sus respectivas especies se presentan en orden alfabético. Los nombres comunes fueron tomados de Robertson \& Allen (2002), Nelson et al. (2004), Love et al. (2005), Rodríguez Romero et al. 2008. Se presenta el nivel trófico, afinidad biogeográfica y las tallas máximas conocidas para las especies.

Las especies registradas en cada una de las áreas, se clasificaron en cinco grupos de tallas siguiendo la propuesta general presentada por Roger \& Ellis (2000), con modificaciones de Gristina et al. (2006). Considerando: I. Pequeños teleósteos (longitud total máxima $<29.9 \mathrm{~cm}$ ); II. Teleósteos medianos (longitud total máxima $\geq 30 \leq 49.9 \mathrm{~cm}$ ); III. Grandes teleósteos (longitud total máxima $\geq 50 \mathrm{~cm}$ ); IV. Especies pequeñas de elasmobranquios (longitud total máxima $\leq 105 \mathrm{~cm}$ ); y V. Especies grandes de elasmobranquios (longitud total máxima $\geq 105.1 \mathrm{~cm}$ ).

\section{RESULTADOS}

Se efectuaron un total de 74 lances prospectivos de $1 \mathrm{~h}$ de duración a diferentes profundidades (11 en septiembre 2004, 30 en febrero y 31 en mayo 2005). La composición específica obtenida durante los cruceros de prospección se conformó por un total de 9898 peces, integrada por dos clases, 15 órdenes, 35 familias, 53 géneros y 70 especies. El orden Perciformes fue el más diverso con 13 familias, 19 géneros y 22 especies. Las familias mejor representadas en número de especies fueron: Paralichthyidae con ocho, Serranidae con seis, Scorpaenidae y Triglidae con cinco especies cada una (Cuadro 1). En el crucero de septiembre del 2004 se registraron 33 especies, en el crucero de

CUADRO 1

Composición de peces demersales que habitan entre 90 y 540 m en el Golfo de California

TABLE 1

Composition of demersal fish that live between 90 and $540 \mathrm{~m}$ in the Gulf of California

\begin{tabular}{|c|c|c|c|c|c|c|c|}
\hline \multirow{2}{*}{\multicolumn{8}{|c|}{$\begin{array}{l}\text { Especie } \\
\text { PHYLUM CHORDATA }\end{array}$}} \\
\hline & & & & & & & \\
\hline \multicolumn{8}{|l|}{ CLASE CHONDRICHTHYES } \\
\hline \multicolumn{8}{|l|}{ ORDEN CHIMAERIFORMES } \\
\hline \multicolumn{8}{|l|}{ FAMILIACHIMAERIDAE } \\
\hline Hydrolagus colliei (Lay y Bennett, 1839) & Quimera manchada & 3.7 & PA-PC & 97 & & & 1 \\
\hline \multicolumn{8}{|l|}{ SUBCLASE ELASMOBRANCHII } \\
\hline \multicolumn{8}{|l|}{ SUBDIVISION SELACHII } \\
\hline \multicolumn{8}{|l|}{ ORDEN CARCHARINIFORMES } \\
\hline \multicolumn{8}{|l|}{ FAMILIA SCYLIORHINIDAE } \\
\hline Apristurus nasutus (Gilbert, 1892) & Pejegato narizon & 3.7 & PA-PS, PP & 69 & & & 1 \\
\hline Galeus piperatus Springer y Wagner, 1966 & Pejegato pimienta & 3.6 & $\mathrm{PC}$ & 30 & & 1 & 1 \\
\hline
\end{tabular}


CUADRO 1 (Continuación)

Composición de peces demersales que habitan entre 90 y 540 m en el Golfo de California

TABLE 1 (Continued)

Composition of demersal fish that live between 90 and $540 \mathrm{~m}$ in the Gulf of California

\begin{tabular}{|c|c|c|c|c|c|c|c|}
\hline Especie & A & $\mathrm{B}$ & $\mathrm{C}$ & $\mathrm{D}$ & $\mathrm{E}$ & $\mathrm{F}$ & G \\
\hline \multicolumn{8}{|l|}{ FAMILIA TRIAKIDAE } \\
\hline Mustelus californicus Gill, 1864 & Cazón mamón & 3.5 & PO-PC & 163 & & & 1 \\
\hline Mustelus henlei (Gill, 1863) & Cazón hilacho & 3.6 & POT & 100 & & 1 & 1 \\
\hline \multicolumn{8}{|l|}{ SUBDIVISION BATOIDEA } \\
\hline \multicolumn{8}{|l|}{ ORDEN RAJIFORMES } \\
\hline \multicolumn{8}{|l|}{ FAMILIA RAJIDAE } \\
\hline Raja inornata Jordan y Gilbert, 1881 & Raya de California & 3.7 & PA-PC & 76 & 1 & 1 & 1 \\
\hline Raja velezi Chirichigno, 1973 & Raya chillona & 3.6 & POT & 83 & 1 & 1 & 1 \\
\hline \multicolumn{8}{|l|}{ ORDEN MYLIOBATIFORMES } \\
\hline \multicolumn{8}{|l|}{ FAMILIA UROLOPHIDAE } \\
\hline Urotrygon rogersi (Jordan y Starks, 1895) & Raya redonda de púas & 3.87 & POT & 46 & 1 & & \\
\hline \multicolumn{8}{|l|}{ CLASE ACTINOPTERYGII } \\
\hline \multicolumn{8}{|l|}{ SUBCLASE NEOPTERYGII } \\
\hline \multicolumn{8}{|l|}{ DIVISION TELEOSTEI } \\
\hline \multicolumn{8}{|l|}{ ORDEN ANGUILLIFORMES } \\
\hline \multicolumn{8}{|l|}{ SUBORDEN CONGROIDEI } \\
\hline \multicolumn{8}{|l|}{ FAMILIA MURAENESOCIDAE } \\
\hline Cynoponticus coniceps (Jordan y Gilbert, 1882) & Congrio espantoso & 4 & POT & 202 & & & 1 \\
\hline \multicolumn{8}{|l|}{ FAMILIA CONGRIDAE } \\
\hline Bathycongrus macrurus (Gilbert, 1891) & Congrio de cabeza corta & 3.7 & POT & 25 & & 1 & \\
\hline \multicolumn{8}{|l|}{ ORDEN ARGENTINIFORMES } \\
\hline \multicolumn{8}{|l|}{ SUBORDEN ARGENTINOIDEI } \\
\hline \multicolumn{8}{|l|}{ FAMILIA ARGENTINIDAE } \\
\hline Argentina sialis Gilbert, 1890 & Argentina del Pacífico & 3.1 & PO-PC & 22 & 1 & 1 & 1 \\
\hline \multicolumn{8}{|l|}{ ORDEN AULOPIFORMES } \\
\hline \multicolumn{8}{|l|}{ SUBORDEN SYNODONTOIDEI } \\
\hline \multicolumn{8}{|l|}{ FAMILIA SYNODONTIDAE } \\
\hline Synodus scituliceps Jordan y Gilbert, 1882 & Chile arpón & 4.5 & PS-PPCH & 64 & 1 & 1 & 1 \\
\hline \multicolumn{8}{|l|}{ ORDEN GADIFORMES } \\
\hline \multicolumn{8}{|l|}{ FAMILIA MACROURIDAE } \\
\hline Caelorinchus scaphopsis (Gilbert, 1890) & Granadero carepala & 3.2 & PO-PC & 34 & 1 & 1 & 1 \\
\hline \multicolumn{8}{|l|}{ FAMILIA MORIDAE } \\
\hline Laemonema verecundum (Jordan y Cramer, 1897) & Morido cabeza grande & 3.3 & PC-PM & 70 & 1 & 1 & 1 \\
\hline Physiculus nematopus Gilbert, 1890 & Carbonero de fango & 3.4 & PS-PP & 29 & 1 & 1 & 1 \\
\hline Physiculus talarae Hildebrand y Barton, 1949 & Carbonero peruano & 3.7 & PS-PP & 25 & & 1 & 1 \\
\hline \multicolumn{8}{|l|}{ FAMILIA MERLUCCIIDAE } \\
\hline Merluccius angustimanus Garman, 1899 & Merluza panameña & 4 & PS-PP & 40 & 1 & 1 & 1 \\
\hline Merluccius productus (Ayres, 1855) & Merluza norteña & 3.6 & PA-PM & 91.5 & 1 & 1 & 1 \\
\hline \multicolumn{8}{|l|}{ ORDEN OPHIDIIFORMES } \\
\hline SUBORDEN OPHIDIOIDEI & & & & & & & \\
\hline FAMILIA OPHIDIIDAE & & & & & & & \\
\hline Cherublemma emmelas (Gilbert, 1890) & Brotula negra & 3.6 & PC-PPCH & 29 & 1 & 1 & 1 \\
\hline
\end{tabular}


CUADRO 1 (Continuación)

Composición de peces demersales que habitan entre 90 y 540 m en el Golfo de California

TABLE 1 (Continued)

Composition of demersal fish that live between 90 and $540 \mathrm{~m}$ in the Gulf of California

\begin{tabular}{|c|c|c|c|c|c|c|c|}
\hline Especie & A & $\mathrm{B}$ & $\mathrm{C}$ & $\mathrm{D}$ & $\mathrm{E}$ & $\mathrm{F}$ & G \\
\hline Lepophidium microlepis (Gilbert, 1890) & Congriperla plateada & 3.6 & POT & 26 & & & 1 \\
\hline Lepophidium negropinna Hildebrand y Barton, 1949 & Congriperla pinta & 3.7 & PS-PP & 52 & & & 1 \\
\hline Lepophidium prorates (Jordan y Bollman, 1890) & Congriperla cornuda & 3.6 & PS-PP & 32 & 1 & 1 & 1 \\
\hline \multicolumn{8}{|l|}{ ORDEN BATRACHOIDIFORMES } \\
\hline \multicolumn{8}{|l|}{ FAMILIA BATRACHOIDIDAE } \\
\hline Porichthys analis Hubbs y Schultz, 1939 & Sapo de luto & 4.1 & PC-PM & 30 & 1 & 1 & 1 \\
\hline \multicolumn{8}{|l|}{ ORDEN LOPHIIFORMES } \\
\hline \multicolumn{8}{|l|}{ SUBORDEN LOPHIOIDEI } \\
\hline \multicolumn{8}{|l|}{ FAMILIA LOPHIIDAE } \\
\hline Lophiodes caulinaris (Garman, 1899) & Rape rabo manchado & 4.5 & PS-PPCH & 40 & & & 1 \\
\hline Lophiodes spilurus (Garman, 1899) & Rape de hebra & 4 & PO-PP & 36 & 1 & 1 & 1 \\
\hline \multicolumn{8}{|l|}{ SUBORDEN LOPHIOIDEI } \\
\hline \multicolumn{8}{|l|}{ FAMILIA LOPHIIDAE } \\
\hline Antennarius avalonis Jordan y Starks, 1907 & Ranisapo antenado & 4 & PS-PPCH & 36 & & 1 & 1 \\
\hline \multicolumn{8}{|l|}{ SUBORDEN OGCOCEPHALIOIDEI } \\
\hline \multicolumn{8}{|l|}{ FAMILIA OGCOCEPHALIDAE } \\
\hline Zalieutes elater (Jordan y Gilbert, 1882) & Murciélago biocelado & 4 & PO-PP & 16.5 & 1 & & 1 \\
\hline \multicolumn{8}{|l|}{ ORDEN SCORPAENIFORMES } \\
\hline \multicolumn{8}{|l|}{ SUBORDEN SCORPAENOIDEI } \\
\hline \multicolumn{8}{|l|}{ FAMILIA SCORPAENIDAE } \\
\hline Pontinus furcirhinus Garman, 1899 & Lapón rojo & 3.8 & POT & 25 & 1 & 1 & \\
\hline Scorpaena guttata Girard, 1854 & ataluña californiano & 3.8 & PO-PC & 47 & & & 1 \\
\hline Sebastes sinensis (Gilbert, 1890) & Rocote boquinegra & 3.5 & $\mathrm{PC}$ & 15 & & & 1 \\
\hline Sebastes spinorbis Chen, 1975 & Rocote ojo espinoso & 3.5 & & & 1 & 1 & \\
\hline Sebastolobus altivelis Gilbert, 1896 & Chancharro espinoso & 3.4 & PA-PC & 39 & 1 & & \\
\hline \multicolumn{8}{|l|}{ ORDEN PLATYCEPHALOIDEI } \\
\hline \multicolumn{8}{|l|}{ FAMILIA TRIGLIDAE } \\
\hline Bellator gymnostethus (Gilbert, 1892) & Vaca enana & 3.4 & PS-PPCH & 15 & 1 & & 1 \\
\hline Bellator xenisma (Jordan y Bollman, 1890) & Vaca doble hocico & 3.3 & PS-PP & 13 & & & 1 \\
\hline Prionotus albirostris Jordan y Bollman, 1890 & Vaca cariblanca & 3.7 & PS-PP & 22 & & & 1 \\
\hline Prionotus ruscarius Gilbert y Starks, 1904 & Vaca rasposa & 3.5 & PC-PPCH & 34 & & 1 & \\
\hline Prionotus stephanophrys Lockington, 1881 & Vaca voladora & 3.5 & $\mathrm{PO}-\mathrm{PPCH}$ & 43 & 1 & & 1 \\
\hline \multicolumn{8}{|l|}{ ORDEN PERCIFORMES } \\
\hline \multicolumn{8}{|l|}{ SUBORDEN PERCOIDEI } \\
\hline \multicolumn{8}{|l|}{ FAMILIA SERRANIDAE } \\
\hline Diplectrum spp. & & 4 & POT & 20 & 1 & 1 & \\
\hline Hemanthias peruanus (Steindachner, 1875) & Cabrilla doblecola & 3.4 & PS-PPCH & 45 & & & 1 \\
\hline Hemanthias signifer (Garman, 1899) & Cabrilla doncella & 3.2 & PS-PP & 42 & & & 1 \\
\hline Paralabrax auroguttatus Walford, 1936 & Cabrilla extranjera & 4.2 & PS-PM & 71 & & 1 & 1 \\
\hline Pronotogrammus eos Gilbert, 1890 & Serrano ojón & 3.5 & POT & 21 & 1 & 1 & \\
\hline Serranus huascarii Steindachner, 1900 & Serrano bandera & 3.6 & POT & 20 & & 1 & \\
\hline
\end{tabular}


CUADRO 1 (Continuación)

Composición de peces demersales que habitan entre 90 y 540 m en el Golfo de California

TABLE 1 (Continued)

Composition of demersal fish that live between 90 and $540 \mathrm{~m}$ in the Gulf of California

\begin{tabular}{|c|c|c|c|c|c|c|c|}
\hline Especie & A & $\mathrm{B}$ & $\mathrm{C}$ & $\mathrm{D}$ & E & $\mathrm{F}$ & G \\
\hline \multicolumn{8}{|l|}{ FAMILIA PRIACANTHIDAE } \\
\hline Pristigenys serrula (Gilbert, 1891) & Ataluña semáforo & 4 & PO-PPCH & 33 & & & 1 \\
\hline \multicolumn{8}{|l|}{ FAMILIA MALACANTHIDAE } \\
\hline Caulolatilus affinis Gill, 1865 & Conejo & 3.8 & PS-PPCH & 50 & 1 & 1 & 1 \\
\hline \multicolumn{8}{|l|}{ FAMILIA CARANGIDAE } \\
\hline Selar crumenophthalmus (Bloch, 1793) & Charrito ojón & 4.1 & $\mathrm{CT}$ & 30 & & 1 & \\
\hline \multicolumn{8}{|l|}{ FAMILIA LUTJANIDAE } \\
\hline Lutjanus guttatus (Steindachner, 1869) & Pargo lunarejo & 4 & PC-PPCH & 80 & & & 1 \\
\hline \multicolumn{8}{|l|}{ FAMILIA HAEMULIDAE } \\
\hline Haemulopsis axillaris (Steindachner, 1869) & Ronco callana & 3.4 & POT & 30 & & 1 & \\
\hline Haemulopsis leuciscus (Günther, 1864) & Ronco ruco & 3.5 & PS-PP & 37 & & 1 & \\
\hline \multicolumn{8}{|l|}{ FAMILIA SCIAENIDAE } \\
\hline Cynoscion phoxocephalus Jordan y Gilbert, 1882 & Corvina picuda & 3.8 & POT & 60 & & & 1 \\
\hline Cynoscion reticulates (Günther, 1864) & Corvina rayada & 3.9 & POT & 90 & 1 & 1 & \\
\hline Isopisthus remifer Jordan y Gilbert, 1882 & Corvina ojona & 4.2 & POT & 35 & & 1 & \\
\hline Micropogonias megalops (Gilbert, 1890) & Chano norteño & 3.3 & $\mathrm{PC}$ & 40 & & 1 & \\
\hline \multicolumn{8}{|l|}{ FAMILIA MULLIDAE } \\
\hline Pseudupeneus grandisquamis (Gill, 1863) & Chivo escamudo & 3.5 & PS-PPCH & 30 & & & 1 \\
\hline \multicolumn{8}{|l|}{ SUBORDEN LABROIDEI } \\
\hline \multicolumn{8}{|l|}{ FAMILIA EMBIOTOCIDAE } \\
\hline Zalembius rosaceus (Jordan y Gilbert, 1880) & Mojarra rosada & 3.3 & PO-PS & 20 & & 1 & 1 \\
\hline \multicolumn{8}{|l|}{ SUBORDEN TRACHINOIDEI } \\
\hline \multicolumn{8}{|l|}{ FAMILIA URANOSCOPIDAE } \\
\hline Kathetostoma averruncus Jordan y Bollman, 1890 & Miracielo buldog & 4.5 & PO-PP & 32 & & 1 & \\
\hline \multicolumn{8}{|l|}{ SUBORDEN CALLIONYMOIDEI } \\
\hline \multicolumn{8}{|l|}{ FAMILIA CALLIONYMIDAE } \\
\hline Synchiropus atrilabiatus (Garman, 1899) & Dragoncillo de asta & 3.5 & PS-PP & 12 & 1 & & \\
\hline \multicolumn{8}{|l|}{ SUBORDEN SCOMBROIDEI } \\
\hline \multicolumn{8}{|l|}{ FAMILIA TRICHIURIDAE } \\
\hline Lepidopus fitchi Rosenblatt y Wilson, 1987 & Pez cinto & 4.1 & PO-PP & 210 & & & 1 \\
\hline Trichiurus nitens Garman, 1899 & Sable del Pacífico & 4.4 & $\mathrm{CT}$ & 234 & & 1 & 1 \\
\hline \multicolumn{8}{|l|}{ SUBORDEN STROMATEOIDEI } \\
\hline \multicolumn{8}{|l|}{ FAMILIA STROMATEIDAE } \\
\hline Peprilus snyderi Gilbert y Starks, 1904 & Palometa salema & 4.1 & PS-PP & 39 & 1 & & 1 \\
\hline \multicolumn{8}{|l|}{ ORDEN PLEURONECTIFORMES } \\
\hline \multicolumn{8}{|l|}{ SUBORDEN PLEURONECTOIDEI } \\
\hline \multicolumn{8}{|l|}{ FAMILIA PARALICHTHYIDAE } \\
\hline Citharichthys fragilis Gilbert, 1890 & Lenguado flaco & 3.5 & PO-PC & 22 & & & 1 \\
\hline Citharichthys gordae Beebe y Tee-Van, 1938 & Lenguado escondido & 3.4 & PC & 14 & & 1 & \\
\hline Etropus peruvianus Hildebrand, 1946 & Lenguado zapatilla & 3.3 & POT & 10 & 1 & 1 & 1 \\
\hline Hippoglossina bollmani Gilbert, 1890 & Lenguado pintado & 3.7 & PS-PP & 20 & 1 & & 1 \\
\hline Hippoglossina stomata Eigenmann y Eigenmann, 1 & Lenguado bocón & 3.7 & PO-PC & 40 & & & 1 \\
\hline
\end{tabular}


CUADRO 1 (Continuación)

Composición de peces demersales que habitan entre 90 y 540 m en el Golfo de California

TABLE 1 (Continued)

Composition of demersal fish that live between 90 and $540 \mathrm{~m}$ in the Gulf of California

\begin{tabular}{|c|c|c|c|c|c|c|c|}
\hline Especie & A & B & $\mathrm{C}$ & $\mathrm{D}$ & $\mathrm{E}$ & $\mathrm{F}$ & G \\
\hline Hippoglossina tetrophthalma (Gilbert, 1890) & Lenguado cuatrojos & 3.6 & PS-PPCH & 40 & & 1 & 1 \\
\hline Paralichthys woolmani Jordan y Williams, 1897 & Lenguado huarache & 4.5 & PS-PPCH & 80 & & 1 & 1 \\
\hline Syacium ovale (Günther, 1864) & Lenguado ovalado & 4 & POT & 25 & & & 1 \\
\hline FAMILIA PLEURONECTIDAE & & & & & & & \\
\hline $\begin{array}{l}\text { Pleuronichthys verticalis Jordan y Gilbert, } 1880 \\
\text { FAMILIA CYNOGLOSSIDAE }\end{array}$ & Platija cornuda & 3.1 & PO-PC & 37 & 1 & 1 & 1 \\
\hline Symphurus leei Jordan y Bollman, 1890 & Lengua colinegra & 3.3 & PM-PP & 15 & 1 & 1 & 1 \\
\hline
\end{tabular}

A: Nombre Común, B: Nivel trófico, C: Provincias y regiones biogeográficas (PA: Provincia Aleutiana, PO: Provincia Oregoniana, PS: Provincia de San Diego, PC: Provincia de Cortés, PM: Provincia Mexicana, PP: Provincia Panámica, PPCH: Provincia Peruano-Chilena, POT: Pacífico Oriental Tropical, CT: Especies Circumtropicales, T: Especies Transpacíficas, AN: Especies Anfiamericanas, D: Talla máxima en centímetros, E: crucero de septiembre 2004 (periodo cálido), F: crucero de febrero 2005 (periodo frío) y G: crucero de mayo 2005 (periodo intermedio).

febrero 47 especies y en el de mayo del 2006 se registraron 49. De las 70 especies registradas 14 presentaron las mayores abundancias en los cruceros realizados; de esas 14 especies destacan por su abundancia relativa y persistencia en los tres cruceros, Argentina sialis (fue abundante en los cruceros de septiembre y mayo),
Caelorinchus scaphopsis (fue abundante en septiembre y mayo) y Cherublemma emmelas (resultó abundante en febrero y mayo) (Cuadro 2). Estas especies son típicos habitantes de la zona profunda dentro del Golfo de California.

La afinidad biogeográfica muestra que dominaron las especies del Pacífico Oriental

CUADRO 2

Listado de especies relevantes según abundancia relativa por crucero exploratorio

TABLE 2

List of relevant species as the relative abundance of exploratory survey

\begin{tabular}{lccc}
\multicolumn{1}{c}{ Especie } & Septiembre & Meses & Mayo \\
Etropus peruvianus & 0.18 & Febrero & 0.001 \\
Argentina sialis & 0.12 & 0.03 & 0.11 \\
Pronotogrammus eos & 0.11 & 0.02 & 0.0 \\
Laemonema verecundum & 0.11 & 0.01 & 0.001 \\
Caelorinchus scaphopsis & 0.08 & 0.18 & 0.17 \\
Physiculus nematopus & 0.06 & 0.04 & 0.002 \\
Pleuronichthys verticalis & 0.05 & 0.04 & 0.002 \\
Porichthys analis & 0.03 & 0.003 & 0.04 \\
Sebastes spinorbis & 0.01 & 0.05 & 0.0 \\
Cherublemma emmelas & 0.002 & 0.14 & 0.12 \\
Merluccius productos & 0.02 & 0.08 & 0.09 \\
Lepophidium prorates & 0.01 & 0.08 & 0.06 \\
Selar crumenophthalmus & 0.0 & 0.06 & 0.0 \\
Citharichthys fragilis & 0.0 & 0.05 & 0.16
\end{tabular}


Tropical (POT), seguidos de las especies de las provincias de San Diego-Panámica; San Diego-Panámica-peruano chilena y provincias Oregoniana-de Cortés respectivamente (Fig. 2).

Se encontró que los peces de profundidad recolectados fueron de tallas pequeñas, del grupo I y II, acumulando $70 \%$ del total, los grupos están compuestos por peces de las familias Ophidiidae, Moridea, Lophidae, Scorpaenidae, Triglidae, Paralichthydae, Pleuronectidae y Cynoglossidae (Fig. 3).

\section{DISCUSIÓN}

La especies registradas en esta región oriental del Golfo de California, corresponde a especies de ecosistemas de escasa exploración que aborda profundidades de hasta $700 \mathrm{~m}$, proporcionando información comparativa con los trabajos previos sobre la ictiofauna de profundidades más allá de los $90 \mathrm{~m}$. De las 70 especies registradas, 14 presentaron las mayores abundancias en los cruceros realizados, destacando Argentina sialis, Caelorinchus scaphopsis y Cherublemma emmelas. El número de especies

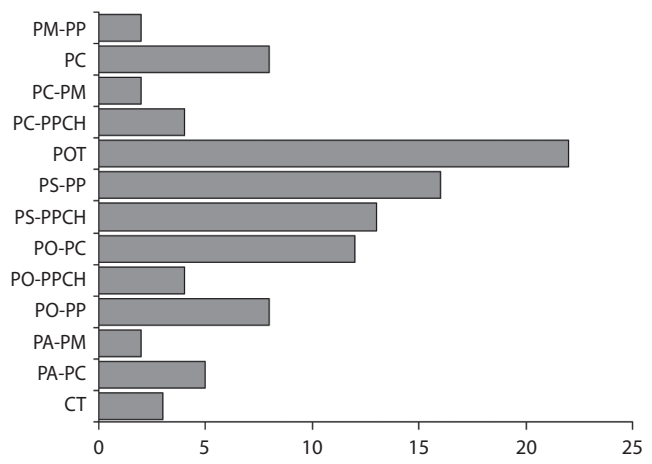

Fig. 2. Porcentajes de afinidad ictiogeográfica (PA: Provincia Aleutiana, PO: Provincia Oregoniana, PS: Provincia de San Diego, PC: Provincia de Cortés, PM: Provincia Mexicana, PP: Provincia Panámica, $\mathrm{PPCH}$ : Provincia Peruano-Chilena; POT: Pacífico Oriental Tropical, CT: Especies Circumtropicales).

Fig. 2. Percentages ichthyogeographic affinity (PA: Aleutian Province, PO: Oregonian, PS: San Diego Province, PC: Cortes Province, PM: Mexican Province, PP: Panamic Province, PPCH: Peruvian-Chilean Province, POT: Tropical Eastern Pacific, TC: Species Circumtropicals). varió dependiendo de la estación del año, 33 en septiembre (verano), 47 en febrero (invierno) y 49 en mayo (primavera). Cabe resaltar que en donde se detectó mayor variación con tendencias más bajas en el número de especies fue en el periodo cálido de septiembre, evento contrario a lo registrado en otras regiones demersales de México, como la costa occidental de Baja California, la cual se distingue por un mayor predominio de especies de afinidad tropical proveniente del Golfo de California (Rodríguez-Romero et al. 2009).

Las especies propias de áreas profundas capturadas en este estudio son la quimera manchada Hydrolagus colliei, el pejegato marrón Apristurus bruneus, el pejegato pimienta Galeus piperatus, el congrio de cabeza corta Bathycongrus macrurus, el morido cabeza grande Laemonema verecundum, el carbonero de fango Physiculus nematopus, el carbonero peruano Physiculus talarae, el rocote boquinegra Sebastes sinensis, el rocote ojo espinoso Sebastes spinorbis y el chancharro espinoso Sebastolobus altivelis, el resto de los miembros de la comunidad forman parte de las capturadas habituales de los barcos camaroneros, hecho por demás interesante, ya que muestra fracciones de las poblaciones no afectadas por los arrastres de pesca, lo que necesariamente debe tener un impacto positivo en la preservación de dichas especies.

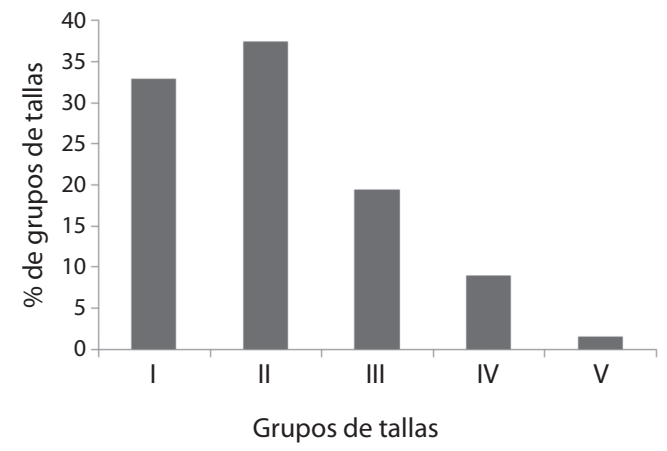

Fig. 3. Porcentaje de los grupos de tallas de los organismos recolectados en la costa oriental del Golfo de California a 90-540m de profundidad.

Fig. 3. Percentage of size groups of organisms collected on the Eastern coast of the Gulf of California to 90-540m depth. 
La mayoría de los peces demersales registrados son especies euritérmicas de amplia distribución en el Pacífico Oriental Tropical. Las especies endémicas del Golfo de California registradas son el rocote ojo espinoso Sebastes spinorbis, el pejegato pimienta G. piperatus, el rocote boquinegra $S$. sinensis, el chano norteño Micropogonias megalops y el lenguado escondido Citharichthys gordae.

El dominio de especies con amplia distribución en la región (POT), es el reflejo de interacción de las masas de agua que conforman la cuenca del Golfo de California, que presenta una clara estratificación, misma que está determinada por la temperatura y la salinidad, dichas especies están distribuidas en diferentes escalas de profundidad (Lavin et al. 1997). Se presentó un total de ocho divisiones zoogeográficas, que marcan un cambio importante en la asociación de las especies de sur a norte. Al norte resaltan las especies de afinidad templada; mientras que en el sur sobresalen las especies típicas del POT como la vaca angelita (Bellator loxias), el sapo de luto (Porichthys analis), el lenguado frentón (Citharichthys platophrys), el lenguado playero (Syacium latifrons), el lenguado zapatilla (Etropus peruvianus), la corvina picuda Cynoscion phoxocephalus y otras. Aspectos similares en estas tendencias fueron documentados por Horn et al. (2006) y Rodríguez Romero et al. (2009). Cabe resaltar, que 17 especies fueron permanentes y el resto presentaron cambios estacionales, resaltando por su abundancia la argentina del Pacífico (Argentina sialis), el morido cabeza grande $(L$. verecundum), la brotula negra (C. emmelas), el congriperla cornuda (Lepophidium proratees) y la merluza (Merluccius productus).

El traslape biogeográfico de la fauna es un reflejo de la posición geográfica del Golfo de California, considerada una zona transicional templado-tropical, en donde se manifiestan especies de afinidades templadas, tropicales y de transición templado-cálido. Las divisiones que caracterizan al Golfo de California (región Norte, Centro y Sur) y los eventos de corrientes, mareas, turbulencias, surgencias y remolinos que se desarrollan en ella, crean una vigorosa circulación, esta circulación interactúa con la profundidad para crear un espectro de energía cinética con periodos bien definidos (Badan-Dangon et al. 1985, López-Martínez 2000), creando eventos de alto dinamismo de la fauna y flora marina, donde los peces aprovechan diversos recursos o eventos para establecerse en zonas de alta productividad biológica, desde zonas someras a profundas (Rodríguez Romero et al. 1998).

Por otra parte, las tallas de los peces recolectados muestran que la mayoría de los organismos son de tamaños pequeños, probablemente debido a la manera en que trabajó la red de pesca (a baja velocidad de arrastre), hecho que pudo permitir una potencial evasión de los organismos de tallas mayores. Se sabe que la composición de las tallas de la captura depende de la velocidad, la abertura de la malla y la selectividad de la red (López-Martínez 2000).

Los estatus taxonómicos en peces con un enfoque de composición, ubicación, caracterización y zoogeografía son fundamentales para desarrollar cualquier tema de biodiversidad y manejo pesquero (Aguilar-Palomino et al. 1996; Rodríguez-Romero et al. 2008). Este estudio aporta información al conocimiento de la fauna íctica demersal de zonas profundas (90 a 540m) de la región oriental del Golfo de California.

\section{AGRADECIMIENTOS}

Estos resultados se derivan del proyecto SAGARPA-CONACYT 2003-002 No. de registro: 024. Se agradece al Centro de Investigaciones Biológicas su apoyo a través del proyecto EP0.12 y a la CONAPESCA la autorización del Permiso de pesca de fomento No. DGOPA/230804. Se agradece al Laboratorio de pesquerías, especialmente a Rufino Morales Azpeitia y Gabriel Rivera Velásquez, se agradece a Xicothencatl Galicia su apoyo logístico. 


\section{RESUMEN}

El Golfo de California tiene una alta variedad de ecosistemas que permiten obtener diferentes servicios y los peces juegan un papel prominente en la ecología, evolución y economía de este. De los peces, las especies costeras han sido reportadas previamente para la mayoría de las zonas litorales, especialmente aquellas especies que son sujetas a explotación pesquera, sin embargo, poco se conoce de las especies que habitan en zonas marinas profundas, debido a lo difícil de su muestreo. Se presenta el listado sistemático y la afinidad biogeográfica de los peces recolectados en tres cruceros de investigación en el Golfo de California con red de arrastre. Se efectuaron 74 lances exploratorios y se capturó un total de 9898 peces, integrados por dos clases, 15 órdenes, 35 familias, 53 géneros y 70 especies. Las familias mejor representadas en número de especies son: Paralichthyidae (ocho especies), Serranidae (seis), Scorpaenidae y Triglidae (cinco cada una). Las familias típicas de aguas profundas son: Ophidiidae, Moridae, Lophiidae, Scorpaenidae, Triglidae, Paralichthydae, Pleuronectidae y Cynoglossidae. El intervalo de tallas encontrado varió de $13 \mathrm{~cm}$ en la vaca doble hocico (Bellator xenisma) a 234cm en el sable del Pacífico Trichurus nitens. La afinidad biogeográfica muestra que dominaron las especies de afinidad al Pacífico Oriental Tropical (POT), seguidos por las especies de las provincias de San Diego-Panámica; San Diego-Panámica-peruano-chilena y provincias Oregoniana-de Cortés respectivamente. Se encontró un traslape biogeográfico de la fauna, la cual es reflejo de la posición geográfica del Golfo de California en los límites de distribución de los elementos en donde se manifiestan especies de afinidades templadas, tropicales y de transición templado-cálido, divisiones que caracterizan al Golfo de California. Los estatus taxonómicos en peces con un enfoque de composición, ubicación, caracterización y zoogeografía son fundamentales para desarrollar cualquier tema de biodiversidad y manejo pesquero.

Palabras clave: Golfo de California, México, peces de profundidad, biogeografía, listado sistemático.

\section{REFERENCIAS}

Abitia Cárdenas, L., R.J. Rodríguez, M.F. Galván, A.J. De la Cruz \& R.H. Chávez. 1994. Lista sistemática de la ictiofauna de Bahía de La Paz, Baja California Sur, México. Cien. Mar. 20: 159-181.

Acevedo Cervantes, A., J. López Martínez, E. Herrera Valdivia \& J. Rodríguez-Romero. 2009. Análisis de la abundancia, dominancia y diversidad de la comunidad de peces demersales de profundidad de 90 a 540 metros en el Golfo de California, México. Interciencia 34: 660-665.
Aguilar-Palomino, B., J. Mariscal-Romero, G. GonzálezSansón \& L.E. Rodríguez-Ibarra. 1996. Ictiofauna demersal de fondos blandos de la plataforma continental de Jalisco y Colima, México, en la primavera de 1995. Cien. Mar. 22: 469-481.

Amezcua-Linares, F. 1985. Recursos potenciales de peces capturados con redes camaroneras en la costa del Pacífico de México, p. 34-94. In A. Yañez-Arancibia (comp.). Recursos potenciales de México: la pesca acompañante del camarón. Programa Universitario de Alimentos, Instituto del Mar y Limnología, Instituto Nacional de la Pesca, UNAM, México.

Arana, P.E., M. Barrios, J. Moraga, A. Mujica \& R. Alarcón. 2002. Evaluación directa del camarón nailon entre la II y VIII regiones, 1999. Informe Final. FIP. 99-08 Universidad Católica del Norte, Coquimbo, Chile.

Badan-Dangon, A., D.J. Koblinsk \& T. Baumgartner. 1985. Spring and summer in the Gulf of California: observation on surface thermal patterns. Oceanol. Acta 8: 13-22.

Boschi, E.E. 2000. Species of decapod crustaceans and their distribution in the American marine zoogeographic provinces. Rev. Inv. y Des. Pesq. 13:7-136.

Brewer, G.D. 1973. Midwater fishes from the Gulf of California and the adjacent Tropical Pacific. Los Angeles County Natural History Museum, Contrib. Sci., 242: 1-47.

Briggs, J.C. 1974. Marine zoogeography. McGraw-Hill, Nueva York, EEUU.

Briggs, J.C. 1995. Global biogeography. Elsevier, Amsterdam, Países Bajos.

Castro-Aguirre, J.L. \& E.F. Balart. 1996. Contribución al conocimiento del origen y las relaciones de la ictiofauna de aguas profundas del Golfo de California, México. Hidrobiológica 6: 67-76.

Castro-Aguirre, J.L. \& H. Espinosa Pérez. 1996. Listados faunísticos de México. VII. Catálogo sistemático de las rayas y especies afines de México (Chondrichthyes: Elasmobranchii: Rajiformes: Batoideiomorpha). Instituto de Biología, UNAM. México D.F., México.

Castro-Aguirre, J.L. 1991. Nuevos registros de peces mesopelágicos y bentónicos en el Golfo de California, México. An. Esc. Nal. Cienc. Biol. Méx. 35: 71-89.

Castro-Aguirre, J.L., E.F. Balart \& J. Arvizu Martínez. 1995. Contribución al conocimiento del origen y 
distribución de la ictiofauna del Golfo de California, México. Hirobiológica 5: 57-78.

Compagno, L.J.V. 1984. Species Catalogue. Sharks of the World. An annotated and illustrated catalogue of sharks species known to date. Part. 2. Carcharhiniformes. FAO Fish. Synop. 4: 251-655.

Chávez, H. \& J. Arvizu-Martínez. 1972. Estudio de los recursos pesqueros demersales del Golfo de California, 1968-1969. III. Fauna de acompañamiento de camarón (peces finos y basura). In J. Carranza (ed.). Mem. VI Congr. Nal. Oceanogr. México, 1969: 361-378.

Chávez, H. 1986. Bibliografía de los peces del Golfo de California. Inv. Mar. CICIMAR, num. esp. 1: 1-267.

De La Cruz-Agüero, J., F.M. Galván, A.C. Abitia, J.R. Rodríguez \& F.J. Gutiérrez. 1994. Lista sistemática de los peces marinos de Bahía Magdalena, Baja California Sur, México. Cien. Mar. 20: 17-31.

Eschmeyer, W.N., E.S. Herald \& H. Hammann. 1983. A field guide to the Pacific coast fishes of North America. Hougthon Mifflin, Boston, EEUU.

Espinosa-Pérez, H., J.L. Castro-Aguirre \& L. Huidobro Campos. 2004. Listados faunísticos de México. IX. Catálogo sistemático de tiburones (Elasmobranchii: Selachimorpha). Instituto de Biología UNAM. D.F., México.

Fischer, W., F. Krupp, W. Schneider, C. Sommer, K.E. Carpenter \& V.H. Niem (eds.). 1995. Guía FAO para la identificación de especies para los fines de pesca. Pacífico Centro-Oriental. FAO. Roma.

Fossen, I., C.F. Cotton, O.A. Bergstad \& J.E. Dyb. 2008. Species composition and distribution patterns of fishes captured by long-lines on the mid-Atlantic ridge. Deep sea Res. II 55: 203-217.

Fujita, T., T. Inada \& Y. Ishito. 1995. Depth gradient structure of the demersal fish community on the continental shelf and upper slope off Sendai Bay, Japan. Mar. Ecol. Prog. Ser. 118: 13-23.

Galván-Magaña, F., L.A. Abitia-Cárdenas, J. RodríguezRomero, H. Pérez-España \& H. Chávez-Ramos. 1996. Lista sistemática de los peces de isla Cerralvo, Baja California Sur, México. Cien. Mar. 22: 295-311.

Galván-Magaña, F., S.F.J. Gutiérrez, C.L.A. Abitia \& J.R. Rodríguez. 2000. The distribution and affinities of the shore fishes of the Baja California Sur Lagoons. In M. Manuwar, S.G. Lawrence, I.F. Manuwar \& D.F. Malley (eds.). Aquatic Ecosystems of México:
Status \& Scoop. Ecovision world monograph series. Backhunys, Holanda.

Garman, S. 1899. Reports on an exploration off the west coast of Mexico, Central America, South America, and off the Galapagos Islands, in charge of Alexander Agassiz, by the U.S. Fish Commission steamer "Albatross" during 1891, Lieut. Commander Z. L. Tanner, U.S.N. commanding. XXVI Pt. 26. The fishes Mem. Mus. Comp. Zool. 24.

Ginsburg, I. 1958. Flounders of the genus Paralichthys and related genera in american waters. U.S. Fish. Wildl. Serv., Fish. Bull. 52: 267-351.

Goode, G.B. \& T.H. Bean. 1895. Oceanic ichthyology: deep-sea and pelagic fishes of the world. Smithsonian Institution.

Gordon, J.D.M., A.O. Bergstad, I. Figueiredo \& G. Menezes. 2003. Deep water fisheries of the Northeast Atlantic: I. Description and current Trends. J. Northw. Atl. Fish. Sci. 31: 137-150.

Grant, A. 2000. Deep sea diversity: Overlooked messages from shallow waters sediments. Mar. Ecol. 21: 97-112.

Grassle, J.F. 1989. Species diversity in deep-sea communities. Trends Ecol. Evol. 4: 12-15.

Grey, M. 1956. The distribution of fishes found below a deep of 2000 meters. Zoology 36: 75-337.

Gristina, M., T. Bahri, F. Fiorentino \& G. Garafalo. 2006. Comparison of demersal fish assemblages in three areas of the Straight of Sicily under different trawling pressure. Fish. Res. 81: 60-71.

Hastings, P.A. 2000. Biogeography of the tropical eastern Pacific: distribution and phylogeny of chaenopsid fishes. Zool. J. Lin. Soc. 128: 319-335.

Horn, M.H., L.G. Allen \& R.N. Lea. 2006. Biogeography. In L.G. Allen, D.J. Pondella \& M.H. Horn (eds.). The ecology of marine fishes: California and adjacent waters. University of California, Berkeley, EEUU.

Jordan, D.S. \& B.W. Evermann. 1896-1900. The fishes of North and Middle America. Bull. Natl. Mus. 47: $1-3313$.

Koslow, J.A., K. Gowlett-Holmes, J.K. Lowry, T. O'Hara, G.C.B. Poore \& A. Williams. 2001. Seamount benthic macrofauna off southern Tasmania: community structure and impacts of trawling. Mar. Ecol. Progr. 213: 111-125. 
López Martínez, J. 2000. Dinámica de la pesquería de camarón café (Penaeus californiensis) en el litoral sonorense y su relación con algunos parámetros océano-atmosféricos. Tesis de Doctorado, Centro Interdisciplinario de Ciencias Marinas, La Paz, Baja California Sur, México.

Love, M.S., C.W. Mecklenburg, T.A. Mecklenburg \& L.K. Thorsteinson. 2005. Resource Inventory of Marine and Estuarine Fishes of the West Coast and Alaska: A Checklist of North Pacific and Arctic Ocean Species from Baja California to the Alaska-Yukon Border. U. S. Department of the Interior, U. S. Geological Survey, Biological Resources Division, Seattle, Washington, EEUU.

McPhail, J.D. 1958. Key to the croakers (Sciaenidae) of the eastern Pacific. Univ. Brit. Columbia, Inst. Fish., Mus. Contrib. 2: 1-20.

Meek, S.E. \& S.F. Hildebrand. 1923-1928. The marine fishes of Panama. Publ. Field. Mus. Nat. Hist., Zool. Ser. 15: 1-1045.

Miller, D.J. \& R.N. Lea. 1972. Guide to the coastal marine fishes of California. Fish Bulletin 157.

Moncayo-Estrada, R., J. Castro-Aguirre \& J. De La CruzAgüero. 2006. Lista sistemática de la ictiofauna de Bahía de Banderas, México. Rev. Mex. Biol. 77: 67-80.

Nelson, J.S., E.J.Crossman, H. Espinosa-Perez, L.T. Findley, C.R. Gilbert, R.N. Lea \& J.D. Williams. 2004. Common and scientific names of fishes from the United States, Canada, and Mexico. American Fisheries Society, Special Publication 29, Bethesda, Maryland.

Nelson, J.S. 2006. Fishes of the world. Wiley, Hoboken, Nueva Jersey, EEUU.

Orr, J.W., M.A. Brown \& D.C. Baker. 2000. Guide to rockfishes (Scorpaenidae) of the genera Sebastes, Sebastolobus, and Adelosebastes of the Northeast Pacific Ocean. NOAA Tech. Mem. NMFS-AFSC-117.

Pérez-España, H., F. Galván-Magaña \& A. Abitia-Cárdenas. 1996. Variaciones temporales y espaciales en la estructura de la comunidad de peces de arrecifes rocosos del suroeste del Golfo de California, México. Cien. Mar. 22: 273-294.

Pérez-Mellado, J. \& L.T. Findley. 1985. Evaluación de la fauna acompañante del camarón comercial capturado en las costas de Sonora y norte de Sinaloa, p. 85-200. In A. Yañez-Arancibia (ed.). Recursos Pesqueros potenciales de México: la pesca acompañante del camarón. Programa Universitario de Alimentos, Instituto del Mar y Limnología, Instituto Nacional de la Pesca, UNAM, México.

Powell, S.M., R.L. Haendrich \& J.D. McEachran. 2003. The deep demersal fish fauna of the Northern Gulf of Mexico. J. Northw. Atl. Fish. Sci. 31: 19-33.

Reyes, P.R. 2007. Peces abisales de la "Triple Unión", lugar de contacto de las placas Antártica, Sudamericana y Nazca (archipielago patagónico noroccidental). Biol. Mar. y Oceanogr. 42: 37-47.

Robertson, D.R. \& G.R. Allen. 2002. Shore fishes of the Tropical Eastern Pacific: an Information System. CD-ROM. Smithsonian Tropical Research Institute, Balboa, Panama.

Robertson, D.R., J.S. Grove \& J.S. McCosker. 2004. Tropical transpacific shore fishes. Pac. Sci. 58: 507-565.

Rodríguez-Romero, J., L.A. Abitia-Cárdenas, F. GalvánMagaña \& H. Chávez-Ramos. 1994. Composición, abundancia y riqueza específica de la ictiofauna de Bahía Concepción, Baja California Sur, México. Cien. Mar. 20: 321-350.

Rodríguez-Romero, J. 1992. Composición, abundancia y riqueza específica de peces de fondos blandos en Bahía Concepción, Baja California Sur, México durante el período Febrero-Septiembre de 1989. Tesis de Maestría, Centro Interdisciplinario de Ciencias Marinas del Instituto Politécnico Nacional (CICIMAR-IPN), La Paz, Baja California Sur, México.

Rodríguez-Romero, J., L.A. Abitia-Cárdenas, F. GalvánMagaña, F.J. Gutiérrez-Sánchez, B. Aguilar-Palomino \& J. Arvizu-Martínez. 1998. Ecology of fish communities from the soft bottoms of Bahia Concepción, México. Arch. Fish. Mar. Res. 46: 61-76.

Rodríguez Romero, J., S. Hernández Vázquez \& J. López Martínez. 2009. Desarrollo potencial de peces desaprovechados. Ciencia y Desarrollo 35: 45-51.

Rodríguez-Romero, J., D.S. Palacios-Salgado, J. LópezMartínez, S. Hernández-Vázquez \& G. Ponce-Díaz. 2008. Composición taxonómica y relaciones zoogeográficas de los peces demersales de la costa occidental de Baja California Sur, México. Rev. Biol. Trop. 56: $1765-1783$.

Rogers, S.I. \& J. Ellis. 2000. Changes in the demersal fish assemblages of British coastal waters during the 20th century. ICES J. Mar. Sci. 57: 866-881. 
Rosenblatt, R.H. \& G.D. Johnson. 1974. Two new species of sea basses of the genus Diplectrum, with a key to the Pacific species. Cal. Fish Game 60: 178-191.

Thomson, D.A. \& N. Mckibbin. 1978. Peces del Golfo de California. Centro de Investigaciones Científicas y Tecnológicas. Universidad de Sonora México, Hermosillo, Sonora, México.

Thomson, D.A., L.T. Findley \& A.N. Kerstitch. 2000 Reef fishes of the Sea of Cortez. University of Texas, Austin, EEUU.
Van Der Heiden, A. 1985. Taxonomía, biología y evaluación de la ictiofauna demersal del Golfo de California, p. 149-200. In A. Yañez-Arancibia (ed.). Recursos Pesqueros potenciales de México: la pesca acompañante del camarón. Programa Universitario de Alimentos, Instituto del Mar y Limnología, Instituto Nacional de la Pesca, UNAM, México.

Walter, H.J. \& R.H. Rosenblatt. 1988. Pacific toadfishes of the genus Porichthys (Batrachoididae) with description of three new species. Copeia: 887-904. 